\title{
POWER CONSUMPTION OF ELECTRODYNAMIC VALVE ACTUATOR
}

\author{
T. Zvolský*
}

\begin{abstract}
The presented analysis deals with a procedure how to compute electric energy consumption of electrodynamic valve actuator of combustion engine. The main aim of the electrodynamic valve control is to ensure independent valve lift and timing. Magnetic field of the actuator was determined using finite element analysis software. The paper also deals with an optimizing of the actuator coil. Forces from the flue-gas acting on valve is included in this model. Valve lift, coil current, voltage and electric energy consumption are graphically shown.
\end{abstract}

Keywords: Valve, Engine, Model, Electrodynamic, Consumption.

\section{Introduction}

Designers strive to achieve the best engine performance. Variable valve control of combustion engine increases engine torque and reduces fuel consumption and emissions. Camless valvetrain can operate independently of the crankshaft of the engine, so the main advantage of the camless valvetrain is to provide independent valve timing. One of the possibilities of the independent valve control is an electrodynamic actuator connected to the valve. Each cylinder valve is actuated by the electrodynamic actuator. Electric energy is required to move the actuator.

\section{Methods}

The basic parts of this actuator are movable coil and stationary permanent magnet, shown in Figure 1. The coil is connected to the valve (Miyoshi, 1999). The coil moves in a cylindrical slot between the pole extensions of the magnetic circuit. The direction of the force can be easily changed by the opposite direction of the coil current. The force of the electrodynamic actuator is independent of the valve stroke. Stiffness of the spring is lower than conventional valve mechanism. The stiffness of the spring is selected to keep the valve closed when the differential pressure of the gases is 1 bar (higher pipe pressure than pressure in engine cylinder).

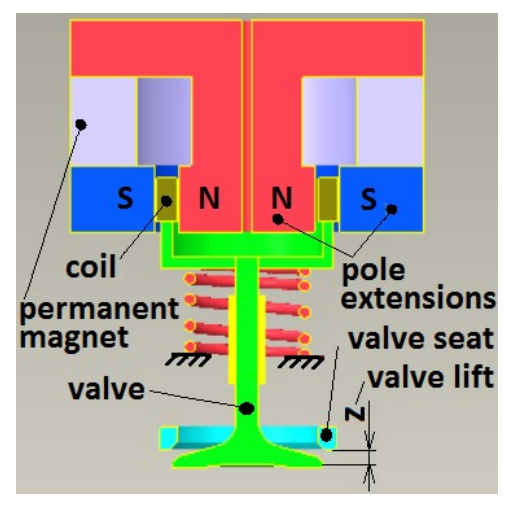

Fig. 1:Electrodynamic actuator

\subsection{Mathematical model of electrodynamic actuator}

The principle of the electrodynamic actuator uses the Lorentz force law (Tumanski, 2011). Lorentz force is the mechanical force acting on a current conducting wire and can be described as.

$$
\vec{F}=I(\vec{\ell} \times \vec{B})
$$

\footnotetext{
Ing. Tomáš Zvolský: Technical University of Liberec, Studentská 2; 461 17, Liberec; CZ, tomas.zvolsky@tul.cz
} 
where $\ell$ is a vector of the length of wire. Direction of the vector $\ell$ is along the wire, aligned with the direction of conventional current flow $I$. If the magnetic field $B$ is perpendicular to the direction of the conductor, the equation of motion is.

$$
F(t)=B \cdot I(t) \cdot \ell=\left(m_{\text {valve }}+m_{\text {coil }}\right) \ddot{z}(t)+k_{\text {spring }} . z(t)+F_{\text {gas }}(t)
$$

From the relationship (2), it is evident, that the force $F$ increases with the increasing conductor length $\ell$. However, with the increasing length of the conductor, it also increases conductor mass, hence the mass of the moving coil $m_{\text {coil }}$. This is disadvantageous because the acceleration of the valve must be quite considerable. Force also increases with increasing current flowing through the coil wire. However, with the increasing current, the energy losses in the conductor increase. The instantaneous conductor power loss $P_{\text {loss }}$ is given by the relationship (3) (Sedlák, 2002).

$$
P_{\text {loss }}(t)=R I^{2}(t)=\frac{1}{\gamma} \frac{\ell}{A} I^{2}(t)
$$

Where $R$ is the resistance of the conductor, which can be determined using by the conductivity $\gamma$, conductor length $\ell$ and conductor cross-sectional area $A$. The optimization therefore consists of finding a length and cross-sectional area of the conductor in which the losses energy for the valve movement will be minimal. The instantaneous conductor power loss can be also expressed using by the coil mass and density of the coil material $\rho$.

$$
P_{\text {loss }}(t)=\frac{1}{\gamma} \frac{\rho \ell^{2}}{m_{\text {coil }}} I^{2}(t)
$$

After expressing the current from equation (2) and inserting into equation (4), we obtain the equation (5) for minimum conductor power loss.

$$
\frac{d P_{\text {loss }}}{d m_{\text {coil }}}=\frac{d}{d m_{\text {coil }}}\left[\frac{\rho}{\gamma B^{2}}\left(\frac{\left(m_{\text {valve }}+m_{\text {coil }}\right)^{2} \ddot{z}^{2}}{m_{\text {coil }}}+\frac{2\left(m_{\text {valve }}+m_{\text {coil }}\right) \ddot{z} K}{m_{\text {coil }}}+\frac{K^{2}}{m_{\text {coil }}}\right)\right]=0
$$

where $K$ is given by the relationship (6).

$$
K=k_{\text {spring }} . z+F_{\text {gas }}
$$

The result of optimization is the relationship (7).

$$
m_{\text {coil }}=\sqrt{m_{\text {valve }}^{2}+\frac{2 m_{\text {valve }} K}{\ddot{z}}+\frac{K^{2}}{\ddot{z}^{2}}}
$$

For intake valve, the spring stiffness $k_{\text {spring }}$ and the gas forces $F_{\text {gas }}$ are negligible. The greatest losses occur at maximum engine speed where the dominant force is the accelerating force. The result of optimization is the relationship (8).

$$
m_{\text {coil }}=m_{\text {valve }}
$$

Energy losses of the actuator for intake valve are the lowest if the coil mass $m_{\text {coil }}$ is identical to the mass of the valve $m_{\text {valve. }}$. It does not matter the cross-sectional area and length of the conductor, only the total mass of the coil is important. Valve mass, including coil connection and spring dynamic mass, is $45 \mathrm{~g}$. Coil mass is also $45 \mathrm{~g}$, so total moving mass is $90 \mathrm{~g}$. Better material for conductor is aluminum than copper. Aluminum has 61 percent of the conductivity of copper, but has only 30 percent of the weight of copper, so the energy losses are reduced by 51 percent. The total resistance of the coil $\mathrm{R}$ is $164 \mathrm{~m} \Omega$. Coil resistance includes the resistance of two unipolar transistors.

\subsection{Permanent magnetic circuit with air gap}

Ansys simulation software was used to find the magnetic field of the actuator. The permanent magnet material is $\mathrm{NdFeB} \mathrm{N} 35$. The results obtained with 3D simulation were further used in the mathematical model. Figure 2 shows the magnetic flux density of the actuator. 


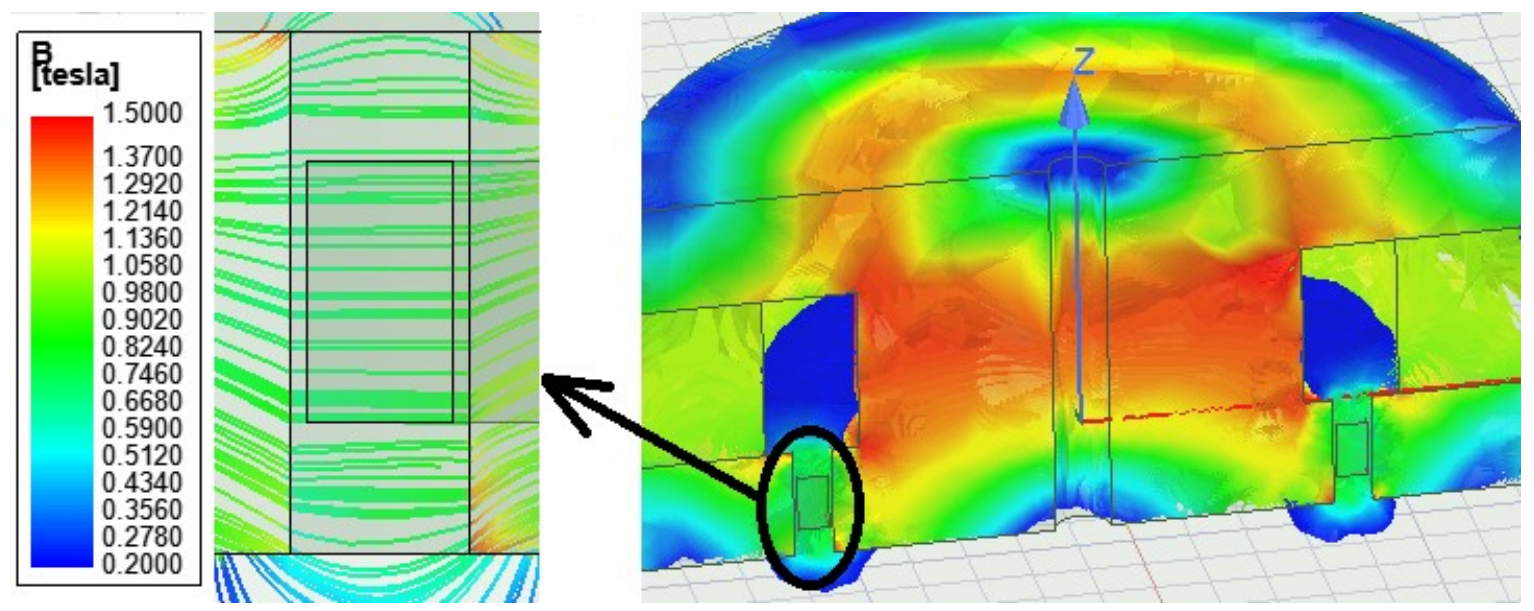

Fig. 2:Magnetic field in the air gap and in the conductor.

Magnetic flux density $B$ is a little bit dependent on the magnitude and direction of the current and position of the coil. Its average value determined from the simulation is $0.74 \mathrm{~T}$. Inductance of the coil $L$ is $519 \mu \mathrm{H}$.

\subsection{Solving the mathematical model}

The coil consists of 30 turns of aluminum wire and the conductor diameter is $1.5 \mathrm{~mm}$. The coil current is given by the equation (9) and can be calculated from the known valve stroke $z(t)$, valve acceleration $\ddot{z}(t)$ and gas forces $F_{\text {gas }}$.

$$
I(t)=\frac{1}{B \cdot \ell}\left[\left(m_{\text {valve }}+m_{\text {coil }}\right) \ddot{z}(t)+k_{\text {spring }} \cdot z(t)+F_{\text {gas }}(t)\right]
$$

Coil voltage $U$ is calculated according to equation (10) (Souček, 2004).

$$
U(t)=B \cdot \ell \cdot \dot{z}(t)+R \cdot I(t)+L \frac{d I(t)}{d t}
$$

Immediate power of the coil $P$ can be calculated from the actual coil voltage and current values. The energy $E$ is obtained according to the relationship (11).

$$
E=\int P(t) d t=\int U(t) I(t) d t
$$

For solving the mathematical model, it was used Matlab software. The graph below shows valve lift, coil voltage, current and input electric energy for the maximal stroke of the intake valve and the exhaust valve per one cycle.

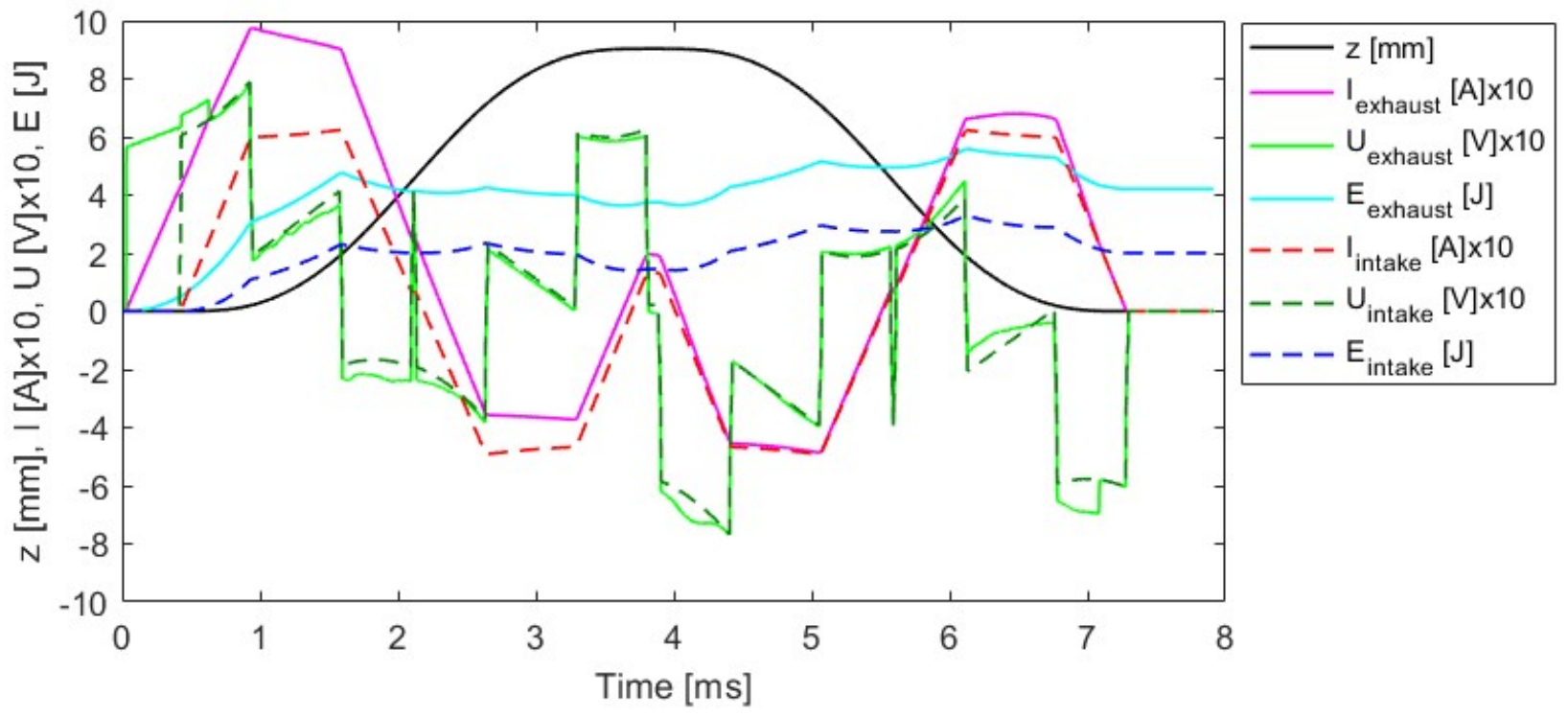

Fig. 3:One working stroke of intake valve (dashed line) and exhaust valve (full line) at 6000 RPM. 
Figure 4 shows the exhaust valve input electric energy per one cycle depending on the engine speed and valve lift for two different coil mass.
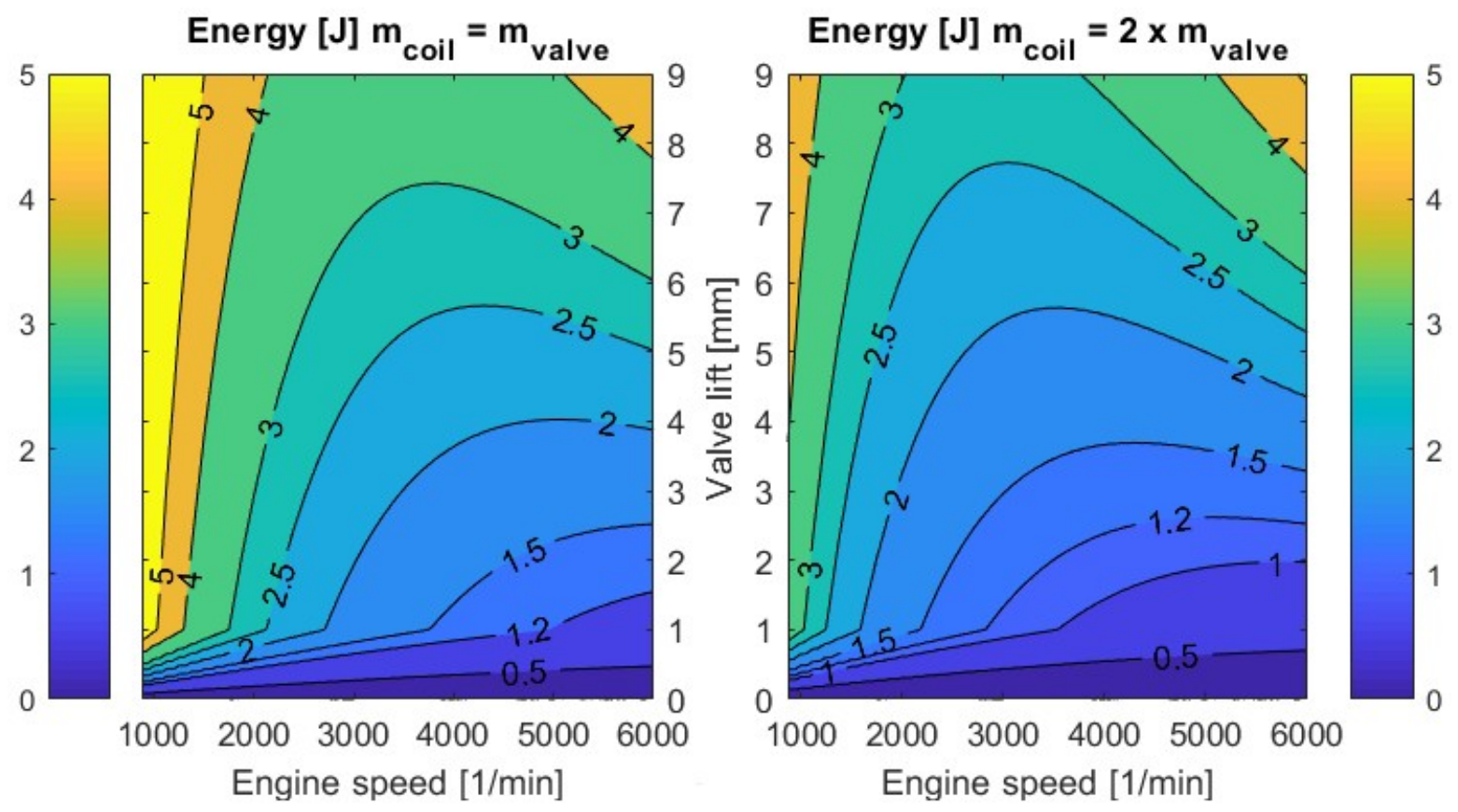

Fig. 4:Input energy for working stroke of the exhaust valve, $m_{\text {coil }}=m_{\text {valve }}$ and $m_{\text {coil }}=2 \times m_{\text {valve. }}$.

If the conductor area extends twice and the coil mass increases by $45 \mathrm{~g}$, the input energy increases by $14 \%$ at 6000 RPM, but at 2000 RPM, the input energy drops by $37 \%$.

\section{Conclusions}

This paper deals with the electrodynamic valve actuator simulation. Magnetic flux density determined from the finite element analysis software is $0.74 \mathrm{~T}$. Finite element analysis software was also used to verify the actuator force. Results obtained using a 3D simulation match mathematical model. The nonlinearity of the actuator force caused by the current is $8 \%$ and non-linearity caused by the coil position is $5 \%$. Input electric energy for the maximal stroke of the intake valve is $2 \mathrm{~J}$ and input electric energy for the maximal stroke of the exhaust valve is $4.2 \mathrm{~J}$ per one cycle at $6000 \mathrm{RPM}$.

\section{Acknowledgement}

This publication was written at the Technical University of Liberec as part of the project 21276 with the support of the Specific University Research Grant, as provided by the Ministry of Education, Youth and Sports of the Czech Republic in the year 2019.

\section{References}

Miyoshi, N., Ohtsubo, K. (1999) Fuji valve: Electric valve drive device in an internal combustion engine, US5983847 Sedlák, B., Štoll, I. (2002) Electricity and magnetism., ISBN 80-200-1004-1, Praha (in Czech).

Souček, P. (2004) Servomechanisms in production machinery. ČVUT, ISBN 80-01-02902-6, Praha (in Czech). Tumanski, S. (2011) Handbook of magnetic measurements. CRC Press, Boca Raton, ISBN 978-1-4398-2951-6. 